\title{
Augmented reality 3D display using head-mounted projectors and transparent retro-reflective screen
}

Shoaib R. Soomro, Hakan Urey

Shoaib R. Soomro, Hakan Urey, "Augmented reality 3D display using headmounted projectors and transparent retro-reflective screen," Proc. SPIE 10126, Advances in Display Technologies VII, 101260E (16 February 2017); doi: $10.1117 / 12.2252439$

SPIE. Event: SPIE OPTO, 2017, San Francisco, California, United States 


\title{
Augmented Reality 3D Display Using Head-Mounted Projectors and Transparent Retro-reflective Screen
}

\author{
Shoaib R. Soomro*, Hakan Urey \\ Koc University, Electrical Engineering Department, Optical Microsystems Laboratory, Istanbul, \\ Turkey
}

\begin{abstract}
A 3D augmented reality display is proposed that can provide glass-free stereo parallax using a highly transparent projection screen. The proposed display is based on a transparent retro-reflective screen and a pair of laser pico projectors placed close to the viewer's head. The retro-reflective screen directs incident light towards its source with little scattering so that each of the viewer's eyes only perceives the content projected by the associated projector. Each projector displays one of the two components (left or right channel) of stereo content. The retro-reflective nature of screen provides high brightness compared to the regular diffused screens. The partially patterned retro-reflective material on clear substrate introduces optical transparency and facilitates the viewer to see the real-world scene on the other side of screen. The working principle and design of the proposed see-through 3D display are presented. A tabletop prototype consisting of an in-house fabricated $60 \times 40 \mathrm{~cm}^{2}$ see-through retro-reflective screen and a pair of 30 lumen pico-projectors with custom 3D printed housings is demonstrated. Geometric calibration between projectors and optimal viewing conditions (eye box size, eye-to-projector distance) are discussed. The display performance is evaluated by measuring the brightness and crosstalk for each eye. The screen provides high brightness (up to $300 \mathrm{~cd} / \mathrm{m}^{2}$ per eye) using 30 lumens mobile projectors while maintaining the $75 \%$ screen transparency. The crosstalk between left and right views is measured as $<10 \%$ at the optimum distance of $125-175 \mathrm{~cm}$, which is within acceptable range.
\end{abstract}

Keywords: 3D displays, Stereoscopic displays, see-throughs screens, retro-reflective surfaces.

\section{INTRODUCTION}

Augmented reality display technologies overlay computer generated digital content on physical objects and allow viewers to perceive and interact with virtual imagery without losing the view of real-world scene. Most of the augmented reality systems use see-through surfaces as transfer screen and one or more image sources to display virtual content. Several techniques have been proposed and demonstrated to design and construct augmented reality displays, most of the them focus on near-to-eye augmented reality displays ${ }^{1-5}$. A typical near-to-eye augmented reality display uses micro display or light projection engine as image source and a holographic-optical element or half-slivered mirror placed close to eye as relay screen ${ }^{2-3}$. The stereo $3 \mathrm{D}$ capability is added by showing different perspective to each eye using separate display sources. The near-to-eye displays can provide quality images without occluding real-world scene, but usually suffer from reduced field of view and involve bulky optical configuration. Various augmented reality systems use headmounted or fixed projectors and distant see-through screens as transfer medium ${ }^{6-9}$, which include the micro-lens array ${ }^{7}$ or resonant nano-particle ${ }^{8}$ based see-through screens. The high brightness gain and personalized display is achieved by using retro-reflective surfaces in the surrounding instead of diffusing screens ${ }^{10-11}$, however to use such surfaces for augmented reality applications requires them to have optical see-through capability and brightness gain.

In this paper, we propose and demonstrate a 3D augmented reality display, which can provide bright stereo 3D while maintaining the higher screen transparency. The proposed display uses a pair of low power laser pico projectors positioned close of viewer's head and a transparent retro-reflective surface as display medium. The retro-reflective characteristics and higher transparency of screen allow the viewer to perceive bright virtual content and real-world scene on the other side of screen seamlessly. We present the concept and design of proposed display, evaluate its performance and determine the optimal viewing conditions based on the retro-reflective characteristics of screen.

*ssoomro13@ku.edu.tr; mems.ku.edu.tr

Advances in Display Technologies VII, edited by Liang-Chy Chien, Tae-Hoon Yoon, Sin-Doo Lee,

Proc. of SPIE Vol. 10126, 101260E - @ 2017 SPIE · CCC code: 0277-786X/17/\$18 · doi: 10.1117/12.2252439 


\section{CONCEPT OF THE DISPLAY}

The proposed display consists of two pico-projectors and a see-through retro-reflective screen as illustrated in Figure 1. A retro-reflective surface reflects the incident light back towards source with narrow scattering and provides brightness gain $^{10}$. The stereo content is projected on a see-through screen using the projector pair placed at distance $d$ from the screen. The baseline distance between the projectors is matched with that of average interpapillary distance (IPD). Each projector illuminates the screen with one of the two channels of a stereo 3D content. Due to the retro-reflective nature of screen, the light from each projector is returned with narrow scattering, and a pair of angular cones is formed at the projectors plane. The width of angular cone and distance from the screen defines the size of eye box for respective eye. When a viewer is standing in front of display such that each eye is positioned within the eye box of corresponding picoprojector, the viewer perceives bright $3 \mathrm{D}$ content on the screen. The transparency of screen is utilized by projecting a stereo content with black background. The high contrast ratio of projectors enable the content to be floating, while at the same time, the real objects on the other side of screen can be clearly seen.

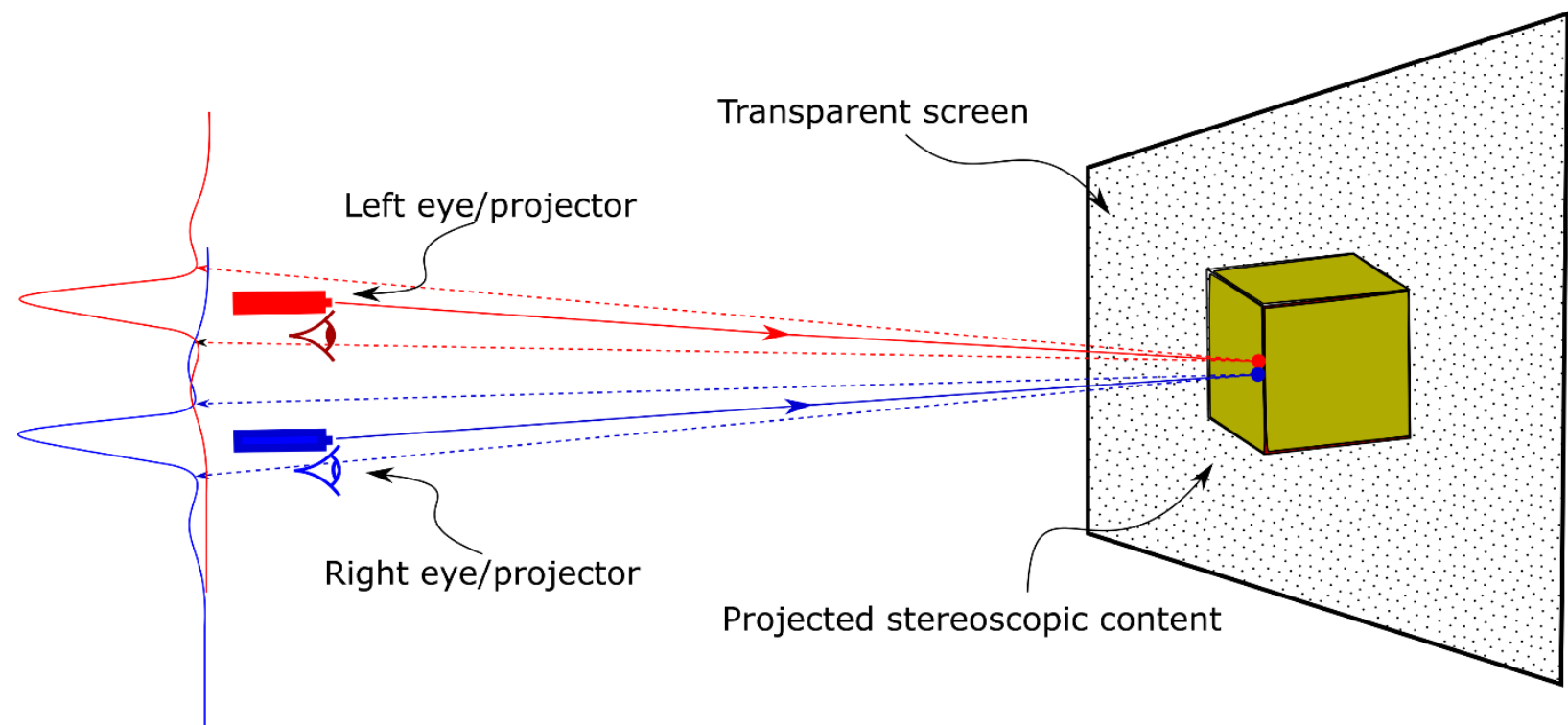

Figure 1: Shows the conceptual illustration of the proposed display, a pair of projectors illuminate the transparent screen with stereo content, where each projector displays separate content to each eye.

We use an in-house designed and fabricated transparent retro-reflective surface as transfer screen. The screen is based on retro-reflective microspheres finely patterned on an optically clear substrate ${ }^{12-13}$. A regular retro-reflective surface provides higher brightness gain around the projector by reflecting major portion of light towards source. We provide the see-through and retro-reflective properties to screen by partially filling a clear substrate using retro-reflective glass microspheres with controlled fill-factor. The microspheres are deposited in shape of micro-patterns such that the individual pattern becomes irresolvable by average human eye when viewed from the working distance. To introduce the enhanced transparency, the retro-reflective fill factor is reduced to $25 \%$ of total screen area to allow retro-reflective projection, while $75 \%$ of area is made see-through.

\section{EXPERIMENTAL SETUP}

The experimental setup was built to demonstrate the 3D augmented reality. The setup consisted a pair of 30 lumen Sony pico-projectors ${ }^{14}$, an in-house developed transparent retro-reflective screen and a pair of 3D printed housings for projectors as shown in Figure 2(a). To minimize the distance between eye and projector, the projectors were oriented vertically and reflective mirrors oriented at $45^{\circ}$ were used to fold the projection light towards screen. The transparent screen was placed within the distance of $\mathrm{d}=50-200 \mathrm{~cm}$ from viewer and the real-objects visible to the viewer through screen were placed on the other side of screen. The horizontal distance between exit pupil of projectors was fixed to $\mathrm{IPD}=6.5 \mathrm{~cm}$ which is equivalent to average interpapillary distance. The minimum gap (s) between eye and projector was $2 \mathrm{~cm}$. 

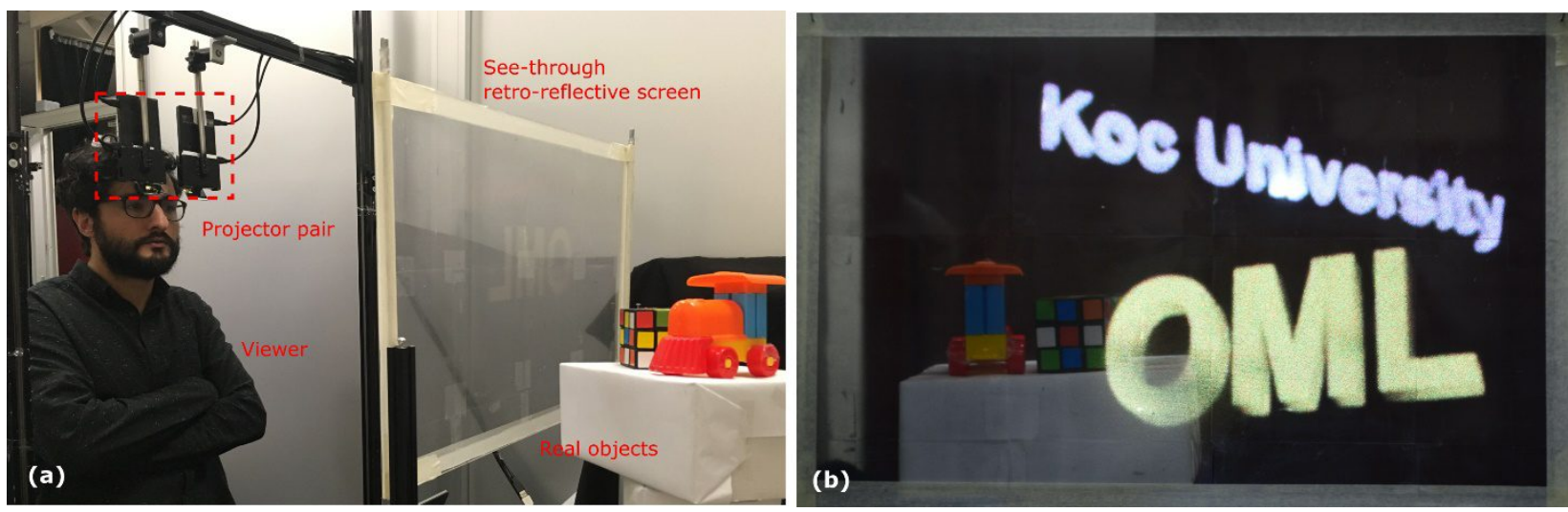

Figure 2: (a) Shows the experimental setup of 3D augmented reality display, the viewer perceives 3D content on see-through screen and, (b) shows the content displayed on screen through one of the projector.

The screen fabrication involved the use of half-shell aluminum coated glass microspheres, where an acrylic sheet with thin adhesive coating was used as base substrate and microspheres were patterned and deposited using a negative shadow mask made of stainless steel. The full-size screen $\left(60 \times 40 \mathrm{~cm}^{2}\right)$ was constructed by tiling together the multiple segments of fabricated screen on clear plexiglass sheet, where size of each screen segment was limited to $16 \times 16 \mathrm{~cm}^{2}$. We used glass microspheres having the refractive index of about $>1.9$ and the mean diameter of $40 \mu \mathrm{m}$. The high refractive index and small size of spheres provided $2^{\circ}$ wide retro-reflective cone ${ }^{12}$. Figure $2(\mathrm{~b})$ shows a content displayed on screen through one of the projector as seen by viewer close to projector. Due to the higher optical transparency of screen, the real objects placed on the other side of screen and a bright projected content can be seen clearly.

\section{RESULTS AND DISCUSSION}

The quality and performance of proposed display was determined by measuring the display brightness and percentage of stereo crosstalk. The properties of display were determined by measuring amount of intensity within retro-reflective angular cone, which is termed as retro-reflective coefficient. We used the experimentally measured retro-reflective coefficient values (as reported in ${ }^{12}$ ) to determine the stereo crosstalk and brightness. The coefficient of retro-reflectivity was measured by using a photometric setup, where a sample of screen was illuminated with $633 \mathrm{~nm}$ collimated laser beam and amount of retro-reflected light as function of angle was measured. Figure 3 shows the measured retroreflective coefficient as function of angle between eye and projector with respect to screen.

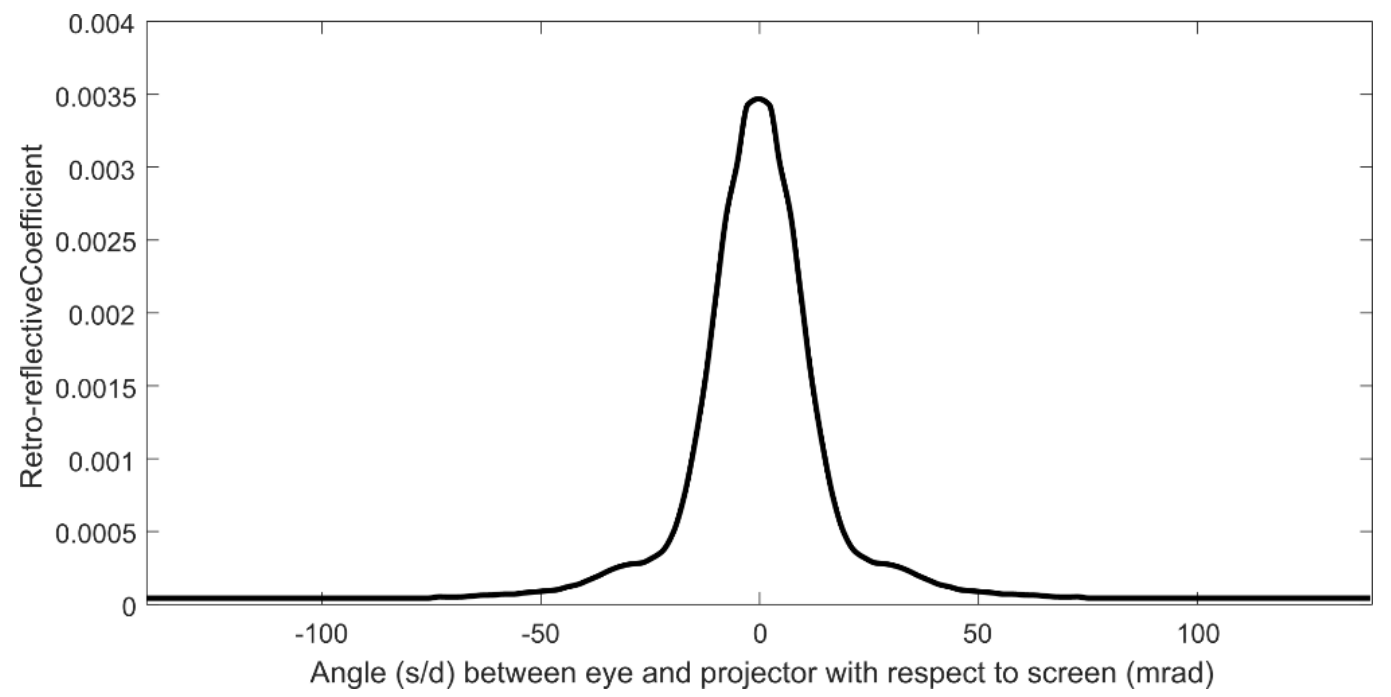

Figure 3:The value of retro-reflective coefficient of screen measured using the photometric setup. The retro-reflective cone provides and angular width of $50 \mathrm{mrad}$. 
The results show maximum coefficient value at $0 \mathrm{mrad}$, while the coefficient value is sharply decreased as the angle between and eye and projector is increased. The figure also shows side lobe which is the result of aperture diffraction due to the small size of microspheres. A constant coefficient value was observed outside the retro-reflective cone, due to the scattering imperfections in screens substrate.

Due to the circular symmetry of retro-reflective cone, the retro-reflective coefficient was measured only in one direction and then rotated symmetrically to obtain the $2 \mathrm{D}$ representation of cone. Since the retro-reflective cone has angular spread, the actual size of the eye box depends on the distance between projector and screen.

The projectors are placed right above eyes with minimum possible gap. The center-center distance between each eye box is equal to IDP, while for each projector, the distance to the closer and farther eye is $\mathrm{s}=2 \mathrm{~cm}$ and $\mathrm{u}=$ $\sqrt{\mathrm{s}^{2}+\mathrm{IPD}^{2}} \mathrm{~cm}$ respectively. For each projector, the eye close perceives high brightness than the farther eye due the higher coefficient value. Considering this configuration and retro-reflective coefficient values, the crosstalk for the left eye can be expressed;

$$
\operatorname{Crosstalk}_{L}(d)=\frac{C_{R}\left(\frac{u}{d}\right)}{C_{L}\left(\frac{s}{d}\right)} 100 \%
$$

Where $C_{R}(u / d)$ is the coefficient value of right projector at distance $u$ from the right projector and $C_{L}(s / d)$ is the coefficient value of left projector at distance $\mathrm{s}$ from left projector. Since the system is symmetrical with identical projector and screen properties, the amount of crosstalk for the left and right eye is same.

The stereo crosstalk for range of viewer-to-screen distance $(\mathrm{d}=50-200 \mathrm{~cm})$ was measured by using the Equation 2 as shown in Figure 4(a). Due to the smaller size of eye box, the display has severe crosstalk (up to $30 \%$ ) at closer distance. The figure shows reduced crosstalk $(<10 \%)$ as the viewer moves at the moderate distance from screen. The eye box overlap increases severely at the longer distance $(>175 \mathrm{~cm})$, which results the increase in crosstalk. The result shows an optimum distance window within $125 \mathrm{~cm}$ and $175 \mathrm{~cm}$ for comfortable stereo experience.
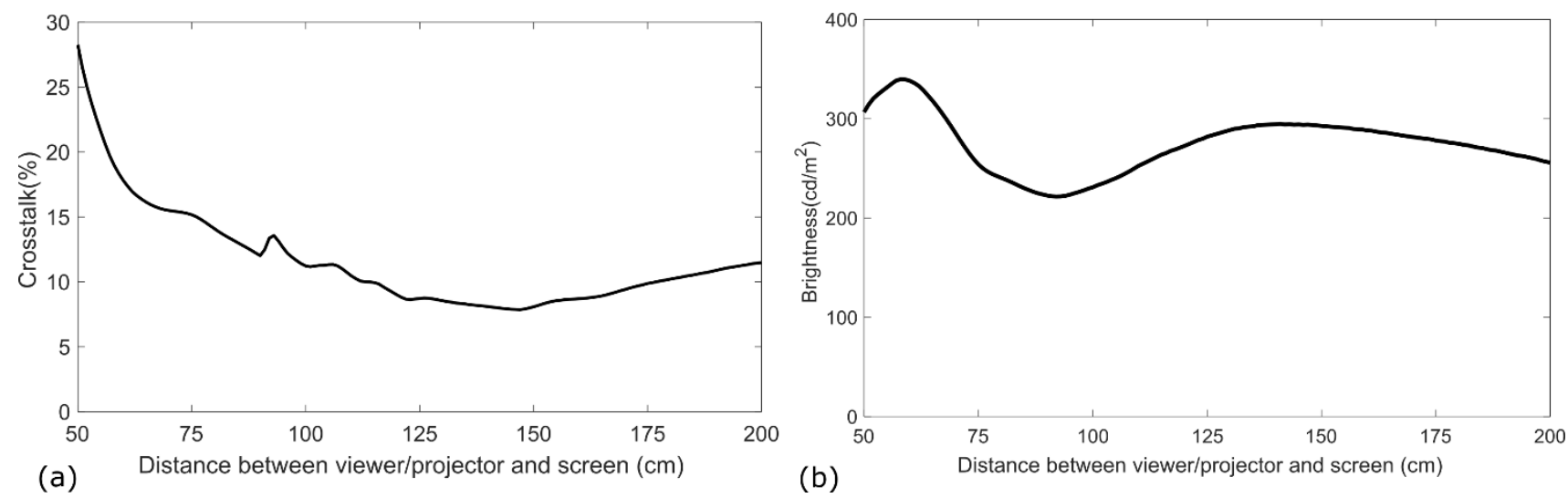

Figure 4: (a) Shows the crosstalk between stereo-channels, and (b) shows the brightness of screen, when screen to viewer distance is changed from $50-200 \mathrm{~cm}$.

The brightness for each eye channel is further calculated by using the experimentally measured retro-reflective coefficient values (Figure 3) and following expression:

$$
B(d)=C\left(\frac{s}{d}\right) \frac{L}{\Psi_{A}}
$$

Where, $\mathrm{C}$ is the value of coefficient at angle s/d, $\mathrm{L}=30$ is the power of pico-projector in lumens, $\Psi$ is the solid angle step size in (determined by detector area used in experiment) and A is the area illuminated by projector. Figure 4(b) shows the brightness perceived by each eye as a function of screen to viewer distance. Significant screen brightness (up to 350 $\mathrm{cd} / \mathrm{m}^{2}$ ) can be perceived at the closer distance due to small projected area. The figure shows relatively consistent brightness as viewer moves away from screen, due to the angular change between eye and projector. The brightness curve also shows a valley at the distance of $90 \mathrm{~cm}$ from screen, which is the result of diffraction lobes in the retroreflective cone. Figure 4(a) and 4(b) collectively shows that the screen can be optimally viewed from distance of 125- 
$175 \mathrm{~cm}$, providing the significant brightness $\left(300 \mathrm{~cd} / \mathrm{m}^{2}\right)$ and minimum crosstalk $(10 \%)$. Figure $5(\mathrm{a})$ and (b) shows the left and right images, when the screen is fixed at the distance of $130 \mathrm{~cm}$ from the projectors/viewer.
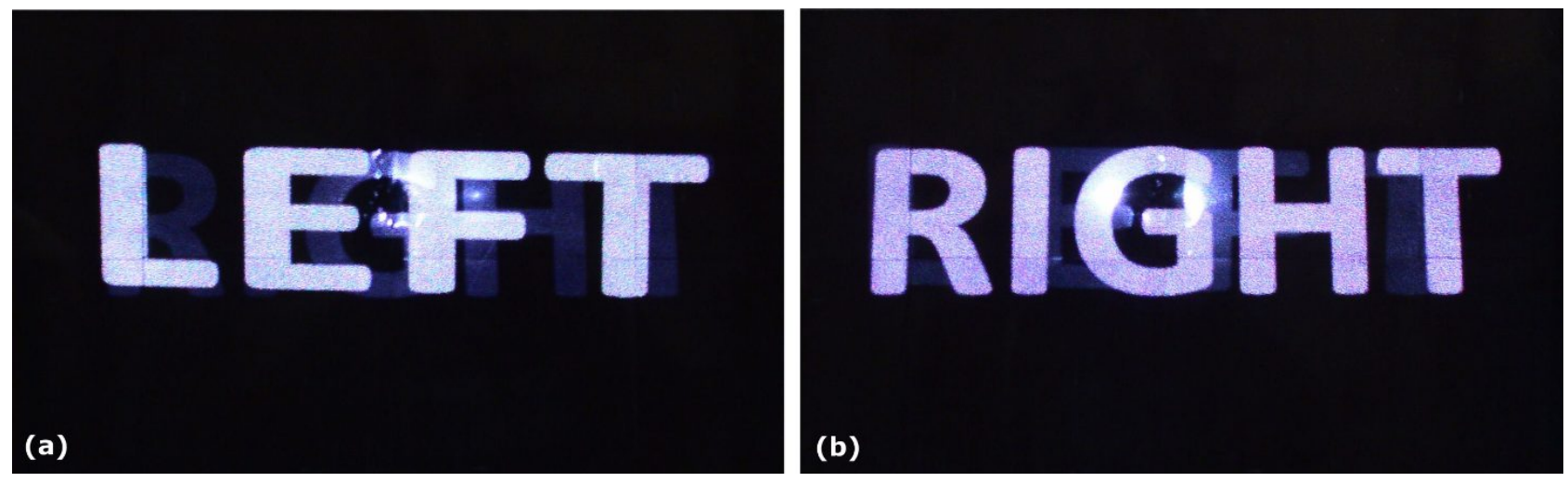

Figure 5: (a) and (b) show the left and right channels of stereo content seen by the viewer at the distance of $130 \mathrm{~cm}$ from screen.

\section{CONCLUSION}

We proposed a stereoscopic 3D augmented reality display based on a transparent retro-reflective screen and a pair of head mounted pico projectors. The high brightness and significant see-through capability was achieved by using a specialized transfer screen based on partially patterned retro-reflective microspheres. Performance of the display was evaluated by determining the retro-reflective properties of screen. The brightness and amount of stereo crosstalk for each eye was estimated by analyzing the size and shape of display eye box for each eye. The prosed display provides the brightness of up to $300 \mathrm{~cd} / \mathrm{m}^{2}$ and $<10 \%$ crosstalk when viewed from $125-175 \mathrm{~cm}$ distance. The working distance of the screen can be further extended by using improved substrate having minimum light diffusion. The crosstalk can be further reduced by polarizing each stereo channel in different orientation or using beam-splitter between eye and projector. The proposed display can be used for variety of augmented reality applications (i.e. Car windshields, museums) to provide the virtual imagery with appropriate depth cues.

\section{ACKNOWLEDGMENTS}

This research is sponsored by European Research Council Advanced Grant (ERC-AdG) Wear3D Project No: 340200. We thank Erdem Ulusoy and Ulas Adiyan for helpful discussions.

\section{REFERENCES}

[1] Zhou, F., Henry, B. and Mark, B., "Trends in augmented reality tracking, interaction and display: A review of ten years of ISMAR", IEEE/ACM International Symposium on Mixed and Augmented Reality", 193-202 (2008).

[2] Microsoft Hololens, https://www.microsoft.com/microsoft-hololens/en-us (accessed December 2016).

[3] Guillaumée, M., Seyed, P.V., Eric, T., Arnaud, M., Gabriel, B., Victor, J. C., Jonas, G., Juergen, B, Randall, S. and Christophe, M., "Curved holographic combiner for color head worn display." J. Disp. Tech. 10(6), 444-449 (2014).

[4] Cakmakci, O. and Jannick. R., "Head-worn displays: a review." J. Disp. Tech. 2(3), 199-216 (2014).

[5] Van, D. N., Tomohiron, M., Kiyoshi, K. and Haruo, T., "Subjective evaluations on perceptual depth of stereo image and effective field of view of a wide-view head mounted projective display with a semi-transparent retro-reflective screen." IEEE International Symposium on Mixed and Augmented Reality (ISMAR), 327-328 (2012).

[6] Ranieri, N., Hagen, S. and Markus, G., "Transparent stereoscopic display and application." Proc. SPIE 9011, Stereoscopic Displays and Applications XXV, 90110P (2014).

[7] Hedili, M.K., Mark O.F. and Urey H., "Transmission characteristics of a bidirectional transparent screen based on reflective microlenses." Opt. Exp. 21(21), 24636-24646 (2012).

[8] Hsu, C.W., Bo, Z., Wenjun, Q., Ofer, S., Brendan, G.D., John, D.J. and Marin, S., "Transparent displays enabled by resonant nanoparticle scattering." Nature communications 5 (2014). 
[9] Soomro, S.R., Ulusoy, E. and Urey, H., "Decoupling of Real and Digital Content in Projection-based Augmented Reality Systems Using Time Multiplexed Image Capture", Journal of Imaging Science and Technology 60 (1), (2017).

[10] Héricz, D, Tamás, S., Viktor, L., Viktor, K. and Pál, K., "Investigation of a 3D head-mounted projection display using retro-reflective screen." Opt. Exp. 22(15), 17823-17829 (2014).

[11] Krum, D.M., Evan, A. S. and Mark, B. "Augmented reality using personal projection and retroreflection." Personal and Ubiquitous Computing 16(1), 17-26 (2012).

[12] Soomro, S.R. and Urey, H., "Design, fabrication and characterization of transparent retro-reflective screen." Opt. Exp. 24(21), 24232-24241 (2016).

[13] Soomro, S.R. and Urey, H., "Retro-reflective Characteristics of Transparent Screen for Head Mounted Projection Displays." OSA Frontiers in Optics, FTu5A-2. (2016).

[14] Sony MP-CL1 mobile projector, http://www.sony.co.in/electronics/projector/mp-cl1 (accessed June 2016). 